\title{
Diagnostic value of echocardiographic markers for diastolic dysfunction and heart failure with preserved ejection fraction
}

\author{
Elisa Dal Canto ${ }^{1,2}$ (D) Sharon Remmelzwaal ${ }^{1} \cdot$ Adriana Johanne van Ballegooijen $^{1,3} \cdot$ M. Louis Handoko $^{4}$. \\ Stephane Heymans ${ }^{5,6,7}$ • Vanessa van Empel ${ }^{5}$ - Walter J. Paulus ${ }^{8} \cdot$ Giel Nijpels $^{2}$ • Petra Elders ${ }^{2} \cdot$ Joline WJ Beulens $^{1,9}$
}

Published online: 2 June 2020

(C) The Author(s) 2020

\begin{abstract}
This study aimed to evaluate the diagnostic performance of echocardiographic markers of heart failure with preserved ejection fraction (HFpEF) and left ventricular diastolic dysfunction (LVDD) in comparison with the gold standard of cardiac catheterization. Diagnosing $\mathrm{HFpEF}$ is challenging, as symptoms are non-specific and often absent at rest. A clear need exists for sensitive echocardiographic markers to diagnose HFpEF. We systematically searched for studies testing the diagnostic value of novel echocardiographic markers for HFpEF and LVDD. Two investigators independently reviewed the studies and assessed the risk of bias. Results were meta-analysed when four or more studies reported a similar diagnostic measure. Of 353 studies, 20 fulfilled the eligibility criteria. The risk of bias was high especially in the patients' selection domain. The highest diagnostic performance was demonstrated by a multivariable model combining echocardiographic, clinical and arterial function markers with an area under the curve of 0.95 (95\% CI, 0.89-0.98). A metaanalysis of four studies indicated a reasonable diagnostic performance for left atrial strain with an AUC of 0.83 (0.70-0.95), a specificity of $93 \%$ (95\% CI, 90-97\%) and a sensitivity of 77\% (95\% CI, 59-96\%). Moreover, the addition of exercise E/e' improved the sensitivity of HFpEF diagnostic algorithms up to $90 \%$, compared with 60 and $34 \%$ of guidelines alone. Despite the heterogeneity of the included studies, this review supported the current multivariable-based approach for the diagnosis of HFpEF and LVDD and showed a potential diagnostic role for exercise echocardiography and left atrial strain. Larger well-designed studies are needed to evaluate the incremental value of novel diagnostic tools to current diagnostic algorithms.
\end{abstract}

Keywords Heart failure with preserved ejection fraction · Diastolic dysfunction · Echocardiography $\cdot$ Systematic review · Meta-analysis

List of abbreviations

$\mathrm{HFpEF} \quad$ Heart failure with preserved ejection fraction
LVEF

NPs
Left ventricular ejection fraction

Natriuretic peptides

Electronic supplementary material The online version of this article (https://doi.org/10.1007/s10741-020-09985-1) contains supplementary material, which is available to authorized users.

Joline WJ Beulens

j.beulens@amsterdamumc.nl

1 Department of Epidemiology and Biostatistics, Amsterdam University Medical Center, Amsterdam, The Netherlands

2 Department of General Practice and Elderly Care Medicine, Amsterdam University Medical Center,

Amsterdam, The Netherlands

3 Department of Nephrology, Amsterdam University Medical Center, Amsterdam, The Netherlands

4 Department of Cardiology, Amsterdam University Medical Center, Amsterdam, The Netherlands
5 Department of Cardiology, CARIM School for Cardiovascular Diseases, Maastricht University Medical Centre,

Maastricht, The Netherlands

6 Department of Cardiovascular Sciences, Centre for Molecular and Vascular Biology, Leuven, KU, Belgium

7 The Netherlands Heart Institute (N1-HI), Utrecht, The Netherlands

8 Department of Physiology, Amsterdam University Medical Center, Amsterdam, The Netherlands

9 Julius Center for Health Sciences and Primary Care, Utrecht University Medical Center, Utrecht, The Netherlands 


$\begin{array}{ll}\text { LAVI } & \text { Left atrial volume index } \\ \text { LVMI } & \text { Left ventricular mass index } \\ \text { LVDD } & \text { Left ventricular diastolic dysfunction } \\ \text { LVFP } & \text { Left ventricular filling pressures } \\ \text { HFrEF } & \text { Heart failure with reduced ejection fraction } \\ \text { STE } & \text { 2-D speckle tracking echocardiography } \\ \text { DST } & \text { Diastolic stress test } \\ \text { QUADAS } & \text { Quality Assessment of Diagnostic Accuracy } \\ & \text { Studies } \\ \text { AUC } & \text { Area under the curve } \\ \text { PH } & \text { Pulmonary hypertension } \\ \text { NCD } & \text { Non-cardiac dyspnoea } \\ \text { AF } & \text { Atrial fibrillation } \\ \text { HFA } & \text { Heart Failure Association } \\ \text { ESC } & \text { European Society of Cardiology } \\ \text { A S E / } & \text { American Society of Echocardiography/ } \\ \text { EACVI } & \text { European Association of Cardiovascular } \\ & \text { Imaging }\end{array}$

\section{Introduction}

Heart failure with preserved ejection fraction (HFpEF) is a complex clinical syndrome associated with high morbidity and mortality, which now accounts for $56 \%$ of the subjects with $\mathrm{HF}$, and its prevalence is increasing [1]. HFpEF is defined by the presence of symptoms and/or signs of HF, a preserved left ventricular (LV) ejection fraction (LVEF, > $50 \%$ ), elevated levels of natriuretic peptides (NPs) and the evidence of cardiac functional and structural alterations underlying HF [2]. Structural alterations include an increased left atrial volume index (LAVI) or left ventricular mass index (LVMI), whereas functional alterations mostly include left ventricular diastolic dysfunction (LVDD). LVDD is defined as the presence of impaired LV relaxation and increased LV chamber stiffness, which increases LV filling pressures (LVFP) [3]. Evidence of LVDD can be obtained invasively through rest or exercise right-sided heart catheterization or non-invasively through echocardiography [2]. There is no single echocardiographic measure that provides evidence of LVDD, but rather a combination of several abnormal indices is recommended to evaluate $\mathrm{LV}$ diastolic function: tissue Doppler indices (E/e' ratio and e' velocities), LAVI and tricuspid regurgitation velocity are the currently recommended variables [3]. However, only a relatively small number of studies validated the use of these echocardiographic indices, showing only a modest correlation with invasive haemodynamic parameters and limited discriminative power [4]. Additionally, the echocardiographic indices proposed by guidelines are normal in 40-75\% of subjects with invasively proven HFpEF [4, 5] and showed lower accuracy in individuals at an early stage of the disease. In fact, these subjects often show a normal or indeterminate diastolic function at resting echocardiography because LVFP are not elevated, or because they vary over time, depending on volume status [6]. Recently, a new stepwise diagnostic approach that includes clinical, laboratory and imaging tests-the HFA-PEFF score-was proposed by the Heart Failure Association (HFA) of the European Society of Cardiology (ESC) with the purpose of integrating novel information into a comprehensive algorithm, in order to better identify subjects with HFpEF at different stages [7]. These recommendations include some of the new techniques that are currently being evaluated as potential diagnostic tools to improve diagnosis and staging of subjects with HFpEF, such as measures of LV deformation by 2D speckle tracking echocardiography (STE) and diastolic stress test (DST)-derived parameters [7]. In addition to these, left atrial (LA) functional parameters such as LA strain recently demonstrated significant correlation with both clinical status and invasive measures of LVFP in subjects with HFpEF and thus could improve HFpEF diagnosis [8]. With this review, we aim to systematically evaluate the diagnostic value of novel echocardiographic indices and multivariable models on accuracy and incremental utility to identify LVDD and HFpEF.

\section{Methods}

\section{Data sources and searchers}

We performed a systematic review of PubMed and EMBASE from their inception to (SR and LS) May 13, 2019, according to the PRISMA-DTA Statement [8]. Search terms included indexed terms from MeSH in PubMed and EMBASE, as well as free-text terms. This search was used for a set of three systematic reviews that describe different types of diagnostic markers for LVDD and HFpEF (NPs, echocardiographic markers and biomarkers). Bibliographies of the identified articles were also hand-searched for relevant publications (see Appendix A). The protocol and search strategy was preregistered on PROSPERO (registration number: CRD42018065018).

\section{Study selection}

Two reviewers independently screened titles, abstracts and full-text articles. Inconsistencies in study selection were resolved through discussion until consensus was reached and, if needed, through the consultation of a third reviewer (SR, EDC, AJvB or JWJB). The eligibility of studies was assessed according to the inclusion and exclusion criteria listed in Supplementary Table 1. 


\section{Quality assessment}

Two reviewers (SR and EDC) independently performed the quality assessment for each study using the QUADAS-2 tool (Quality Assessment of Diagnostic Accuracy Studies) [9]. QUADAS-2 consists of four domains: patient selection, index test, reference standard and flow and timing. Quality is assessed in each domain to estimate risk of bias and concerns regarding applicability. The patient selection domain assessed whether the selection of participants could have introduced bias. The index test and reference standard domains assessed whether the conduct or interpretation of the index test and reference standard, respectively, may have introduced bias. The flow and timing domain addressed the time interval between index test and reference standard (9). Any discrepancies or disagreements between the authors were resolved through discussion until consensus was reached and, if needed, through the consultation of a third reviewer (JWJB).

\section{Diagnostic performance and data extraction}

Two authors (SR and EDC) extracted data independently, according to a standard protocol that included first author, year of publication, country, journal, study design, markers (echocardiographic \pm clinical/laboratory parameters), outcome measures, population description, reference diagnosis and measures of diagnostic performance.

\section{Data synthesis}

Study characteristics of the studies were described in a systematic manner according to the diagnostic markers. Studies were meta-analysed using a random-effects model when three or more studies investigated the same diagnostic measure for the same echocardiographic marker in a similar study population and with a similar control population. In addition, the studies had to provide confidence intervals $(95 \% \mathrm{CI})$ of this diagnostic performance measure or sufficient information $(2 \times 2$ table $)$ to compute these confidence intervals. Forest plots of random-effects meta-analyses were fitted for AUCs, sensitivities and specificities. Heterogeneity was tested using $I^{2}$, where an $I^{2}>75 \%$ is considered as substantial heterogeneity. All analyses and plots were performed in RStudio version 3.4.2 using the metafor package [10].

\section{Results}

\section{Search results}

We screened 11,727 titles, which yielded 353 potentially relevant studies. In total, 20 studies met the inclusion criteria.
The remainder was excluded according to the criteria listed in the PRISMA flowchart (see Supplementary Fig. 1).

\section{Quality assessment}

The QUADAS-2 domain with the highest proportion of high risk of bias was patient selection (Supplementary Fig. 2) with 13 studies (65\%) demonstrating a high risk of bias mostly due to case-control design or to a non-consecutive or non-random inclusion of subjects. In the other three of the four QUADAS2 domains (index test, reference test and flow and timing), eight (40\%), three (15\%) and eight (40\%) studies, respectively, demonstrated a high of risk of bias. In the QUADAS-2 domain reference standard, 12 studies $(60 \%)$ showed an unclear risk of bias. On the other hand, most of the studies showed low concerns regarding applicability with the highest proportion of high concerns for the reference standard domain (six studies, $30 \%$ ). None of the studies was excluded based on the quality assessment.

\section{Study characteristics}

Of the 20 included studies, ten were performed in the USA, seven in Europe, two in Japan and one in Australia (Table 1). Eighteen studies (90\%) were published in the past 10 years (2009-2019). Sixteen were cross-sectional and four were case-control studies performed in subjects referred for right- and/or left-sided heart catheterization. As clinical outcome, 13 studies used HFpEF [5, 11-22], two used HFpEF with associated pulmonary hypertension $(\mathrm{PH}) \quad$ [23, 24], one used "early" HFpEF [25], and four used LVDD [26-29]. The reference diagnosis always included the echocardiographic evidence of a normal LVEF and one or more invasive measures of elevated LVFP (LV end-diastolic pressure or pulmonary capillary wedge pressure), impaired LV relaxation (isovolumetric relaxation time or constant $\tau$ ) and increased LV stiffness (LV stiffness constant $b$ ). Conventional transthoracic rest echocardiography was the most commonly used index measure $(n=10)$ followed by STE $(n=8)$ and by DST $(n=2)$. As echocardiographic predictor, seven studies used a combination of echocardiographic markers or multivariable models that included also demographics, medications, biochemical and arterial function parameters; eight studies used LV and LA strain parameters; and two studies used DST data and three of them used single standard echocardiographic parameters.

\section{Measures of diagnostic performance: HFpEF}

\section{Multivariable models}

In general, multivariable predictors showed good diagnostic performance (Table 2). The highest diagnostic performance was demonstrated by a combination of echocardiography 


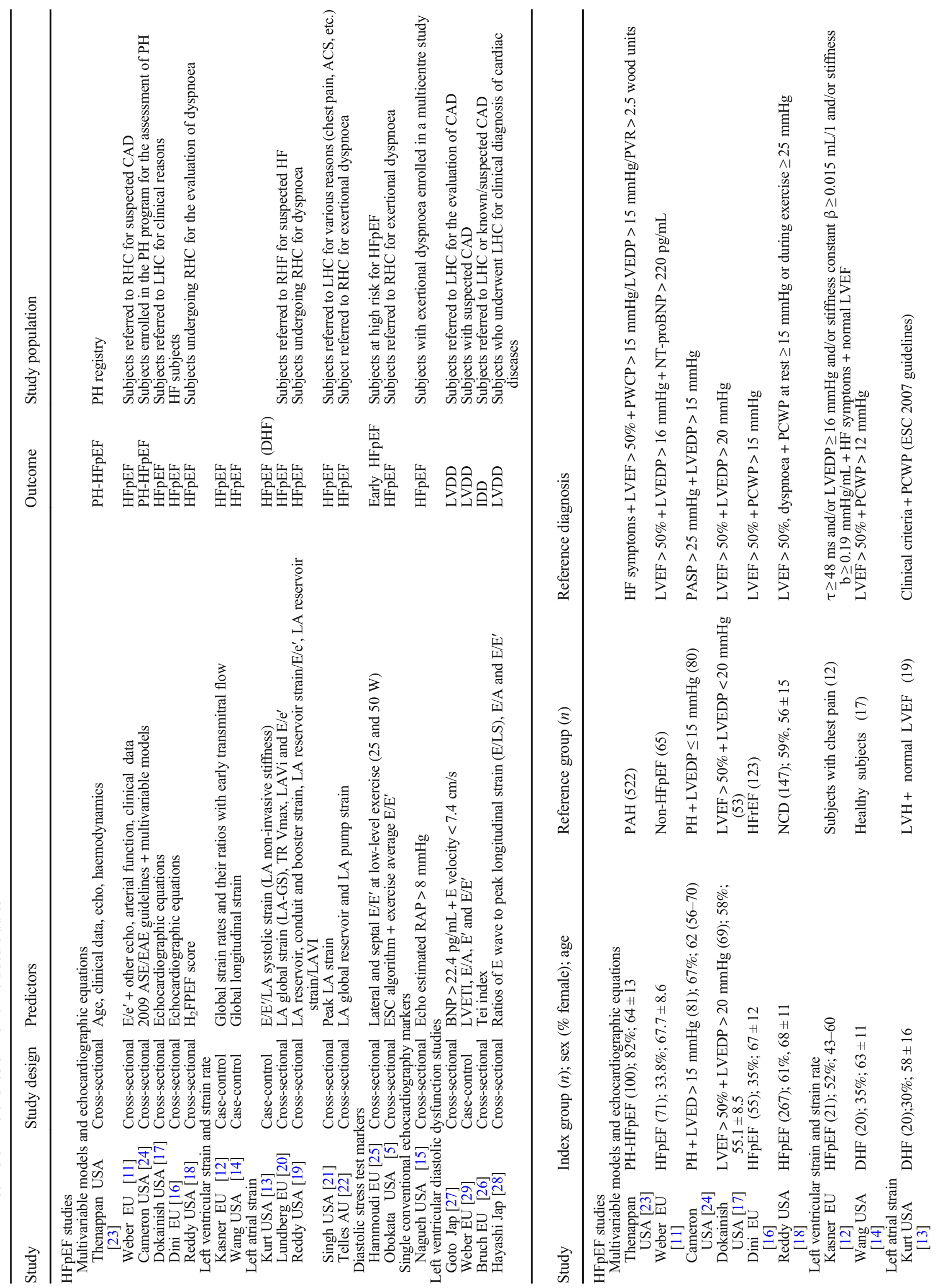




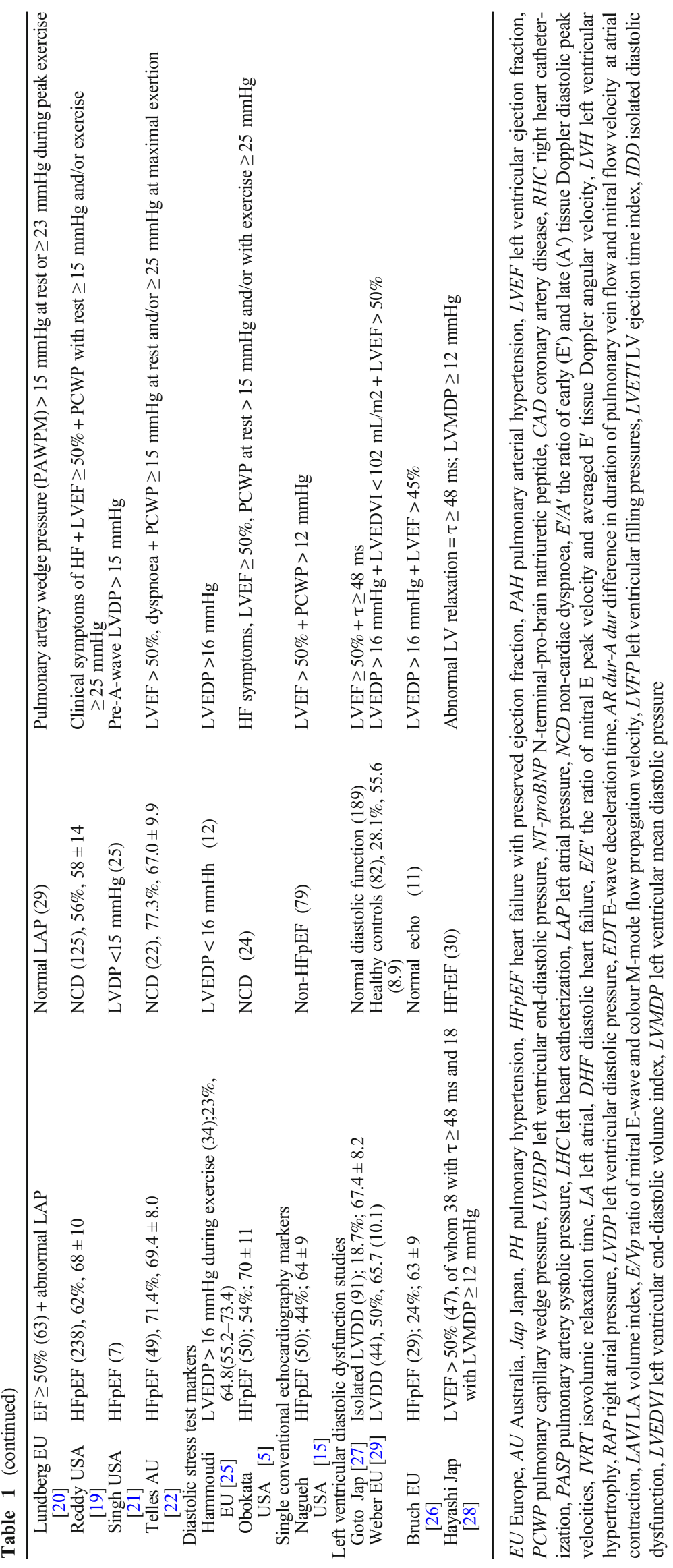


and pulsatile arterial function data with an AUC $=0.95(95 \%$ CI, 0.89-0.98). The addition of aortic pulse pressure to echocardiographic and clinical markers led to a highly significant net reclassification index of up to $33 \%$ and reduced the number of undiagnosed HFpEF subjects from 60 to 24 [11]. The $\mathrm{H}_{2}$ FPEF score showed a very good diagnostic performance to estimate the likelihood of HFpEF among subjects with unexplained dyspnoea [18]. The $\mathrm{H}_{2} \mathrm{FPEF}$ score is based on four clinical characteristics (body mass index, anti-hypertensive medications, atrial fibrillation [AF] and age) and two echocardiographic markers (E/e' and pulmonary artery systolic pressure) and provided good discrimination of HFpEF from subjects with non-cardiac dyspnoea $(\mathrm{NCD})(\mathrm{AUC}=0.84,0.80$ 0.88 ). The performance was maintained in the independent validation cohort with an $\mathrm{AUC}=0.87(0.79-0.94)$ [18].

\section{Meta-analyses on LA strain}

The utilization of LA strain indicated high diagnostic performance without clinical or laboratory data. LA global or reservoir or peak strain was most commonly tested [19-22] with the addition of conduit and booster strain $[19,22]$ and of indirect measures of LA compliance (LA strain/E/e') [19] and LA stiffness (E/e'/LA strain) [13, 22]. The best diagnostic ability was demonstrated by LA global strain for detecting elevated LVFP both at rest $(\mathrm{AUC}=0.87)$ and during exercise $(\mathrm{AUC}=0.93)$ in subjects with HF symptoms, outperforming conventional echocardiographic markers such as E/e' (delta AUC +0.19 during rest and +0.37 during stress) and LAVI (delta AUC +0.08 during rest and +0.27 during stress) [20] Four studies reported sensitivity and specificity for LA global strain with a mean of $77 \%\left(59-96 \% ; I^{2}=93.7 \%\right)$ and $93 \%$ $\left(90-97 \% ; I^{2}=0.22 \%\right)$, respectively, and three studies reported AUCs with a mean of $0.83\left(0.70-0.95, I^{2}=88.3\right)$ (Fig. 1). The high heterogeneity as shown by the meta-analysis for sensitivity and AUC can be explained by the broad range of values observed among the included studies, which for sensitivity ranged from 56 to $92 \%$ and for AUC from 0.72 to 0.93 and by the small sample sizes. On the other hand, all the included studies showed a high ability of LA strain to rule out HFpEF and thus a high specificity with low heterogeneity.

\section{Diastolic stress test}

Two studies evaluated the role of DST in the diagnosis of HFpEF. The first one found that $\mathrm{E} / \mathrm{e}^{\prime}$ at low-level exercise was valuable for predicting abnormal LVFP with a sensitivity of $90 \%$ but only in subjects with cardiac disease [25]. The second study evaluated the incremental utility of DST to the diagnostic approaches proposed by ESC and American Society of Echocardiography/European Society of Cardiovascular Imaging (ASE/EACVI) to diagnose HFpEF: the addition of exercise $\mathrm{E} / \mathrm{e}^{\prime}$ to the ESC and ASE/EACVI
2016 proposed algorithm indicated a much higher sensitivity compared with either of them alone (90 versus 60 and 34\%, respectively) [5].

\section{Measures of diagnostic performance: diastolic dysfunction}

Five studies investigated echocardiographic markers for the detection of LVDD. The best diagnostic performance was demonstrated by the ratio of $\mathrm{E}$ wave to peak longitudinal strain $(\mathrm{E} / \mathrm{LS})$ to predict elevated LVFP in a population of subjects with suspected cardiac disease (AUC $=0.86$ versus 0.74 of $\left.E / e^{\prime}\right)[28]$.

\section{Discussion}

Since HFpEF is the predominant form of HF [1], the detection of this condition gained considerable interest. Standard resting echocardiography has still a pivotal role in the detection of $\mathrm{HFpEF}$, but it provides only indirect evidence of pressurevolume relationships, and it might leave a significant proportion of subjects undetected. In this systematic review, a large variety of echocardiographic markers were investigated and yielded variable results for the diagnostic performance. The main findings are as follows: (1) multivariable models including clinical, echocardiographic and possibly arterial function variables demonstrated the best diagnostic performance. (2) LA strain may provide good discrimination capacity of HFpEF subjects and enhanced diagnostic accuracy beyond conventional echocardiographic measures. (3) Addition of exercise $\mathrm{E} / \mathrm{e}^{\prime}$ to resting echocardiography findings improves HFpEF diagnosis.

\section{Multivariable models}

As expected, multivariable models demonstrated the best diagnostic performance, along the lines of what current guidelines recommend to use in clinical practice for the diagnosis of HFpEF. This can be explained by the complex pathophysiology of HFpEF, which is driven by advanced age and comorbidities, and caused by the interplay of multiple impairments in LV diastolic and systolic function, chronotropic reserve, arterial-ventricular mismatching, vascular and endothelial dysfunction, pulmonary hypertension and impaired systemic vasodilator reserve $[30,31]$. Therefore, a multivariable algorithm that provides integrated information on all these aspects is necessary to evaluate diastolic function. Among the included studies, the highest diagnostic accuracy was demonstrated by a multivariable model combining clinical and echocardiographic markers with arterial function measures, thereby demonstrating that measures of pulsatile arterial haemodynamics may complement echocardiography for the diagnosis of 
HFpEF $[11,31]$. Another combination of clinical and echocardiographic markers that provided a better discrimination of HFpEF from NCD than currently used diagnostic algorithms is the $\mathrm{H}_{2}$ FPEF score, with a delta AUC of $+0.17(0.12-0.22)$ in the derivation cohort and a delta AUC of $+0.21(0.10-0.31)$ in the test cohort versus 2016 ESC guidelines [18]. However, external validation, which is a crucial step before introducing a new diagnostic model in clinical practice, was not performed. Overall, none of the included studies performed external validation, and only three performed validation in separate groups of subjects belonging to the same research centre $[16,18,21]$. Recently, the $\mathrm{H}_{2} \mathrm{FPEF}$ score was validated in the Alberta HEART population, showing a sensitivity of $90 \%$ of a score $>2$ to detect HFpEF and a specificity of $82 \%$ of a score $<6$ to rule out HFpEF [32]. Despite these promising results, the $\mathrm{H}_{2} \mathrm{FPEF}$ score still requires further validation and refinement.

\section{Left atrial strain}

The left atrium plays a key role in HFpEF pathophysiology, and indices of LA mechanics have diagnostic and prognostic utility in HFpEF [33]. STE can assess LA function, remodelling and distensibility, and LA strain can impair independently of LA size [33]. Five recent cross-sectional studies demonstrated the ability of LA strain to correctly classify dyspnoeic subjects as HFpEF with superior sensitivity and specificity than standard echocardiographic parameters $[13,19,22]$ or to identify elevated LVFP more accurately than guidelines $[20,21]$. Specifically, LA reservoir strain enabled to identify HFpEF from NCD with an AUC $=0.72(0.66-0.77)$, outperforming other commonly used indices of diastolic function $[19,20]$. Similarly, LA global strain managed to detect elevated LVFP both at rest and during exercise (AUCs $=0.87$ and 0.93 , respectively) and showed a better agreement with invasively determined LVFP than ESC 2016 guidelines (91 versus $81 \%$ ) [21]. Among the studies that tested novel indices combining LA strain with Doppler measures of LV pressures, LA non-invasive stiffness showed the highest diagnostic performance in distinguishing subjects with $\mathrm{HFpEF}$ from those with LVDD, with an AUC $=0.85$ (0.72-0.98) [13]. The meta-analysis of four studies indicated a very high specificity (93\%) of LA global strain, in combination with a nonsignificant heterogeneity $\left(I^{2}\right.$ of approximately $\left.0 \%\right)$ and a good sensitivity $(77 \%)$ although with consistent heterogeneity $\left(I^{2}>\right.$ $90 \%$ ), which indicate a high ability of LA strain to rule out HFpEF when normal, and a variable capacity to diagnose HFpEF when abnormal. The meta-analysis of three studies indicated also a good ability of LA strain to predict HFpEF diagnosis with an AUC of 0.83 , although with significant heterogeneity $\left(I^{2}\right.$ of $\left.88 \%\right)$. Altogether, these results suggest a potential usefulness of LA strain in the non-invasive diagnostic evaluation of HFpEF. However, the studies that evaluated the diagnostic performance of LA strain established different optimal cut-off values for the identification of HFpEF subjects, ranging from -32.3 to $-20 \%$, and therefore, further studies are warranted to establish a definitive cut-off for abnormal LA strain. Additionally, it should be noted that STE is not routinely available worldwide and requires postprocessing time, which questions its diagnostic utility in clinical practice for non-academic centres.

\section{Diastolic stress test}

Another imaging test with a potential diagnostic role in the diagnosis of HFpEF is the DST. Both ESC and ASE/EACVI guidelines already recommended to perform DST when resting echocardiography does not explain the symptoms of HF, especially when dyspnoea is present only with exertion $[2,3]$. Recently, the DST has been integrated in the new HFA diagnostic recommendations, as part of the advanced HFpEF workup, to be performed if a subject who already underwent clinical, biomarkers and resting echocardiography assessment has an intermediate HFA-PEFF score [7]. The utility of exercise data is clearly evident on top of resting echocardiographic data, as the utilization of exercise E/e' alone (>14) indeed significantly improved the sensitivity of the diagnostic workup to $90 \%$ compared with $60 \%$ of ESC guidelines [5]. Addition of exercise $\mathrm{E} / \mathrm{e}^{\prime}$ also improved classification beyond the resting ESC criteria, with a negative predictive value of 87 versus $83 \%$ [5]. Hence, our results confirm the utility of DST not only to identify HFpEF in euvolemic subjects with inconclusive resting echocardiography but also to rule out HFpEF, when unequivocally normal. However, we must point out that the feasibility and the quality of echocardiographic measures decrease during exercise; for instance, tricuspid regurgitation velocity was measureable only in $49 \%$ of subjects at peak exercise [5]. Moreover, although a low-level exercise test with stepwise increase of the workload is recommended for the DST, there is no universally adopted protocol at the moment.

\section{Strengths and limitations}

To our knowledge, this is the first systematic review on novel echocardiographic markers for HFpEF and LVDD including a meta-analysis. Multiple databases were extensively searched, and article selection, data extraction and quality assessment were performed in duplicate according to a standardized protocol. Moreover, no geographical differences were detected, which increases the generalizability of the results. The review findings were limited by the heterogeneity and the quality of the included studies, which applied to the study design (case control versus cross-sectional), the study population (subjects with unexplained dyspnoea versus subjects with suspected coronary artery diseases), the reference standard (different invasive measures with different cut-off values) and the index 


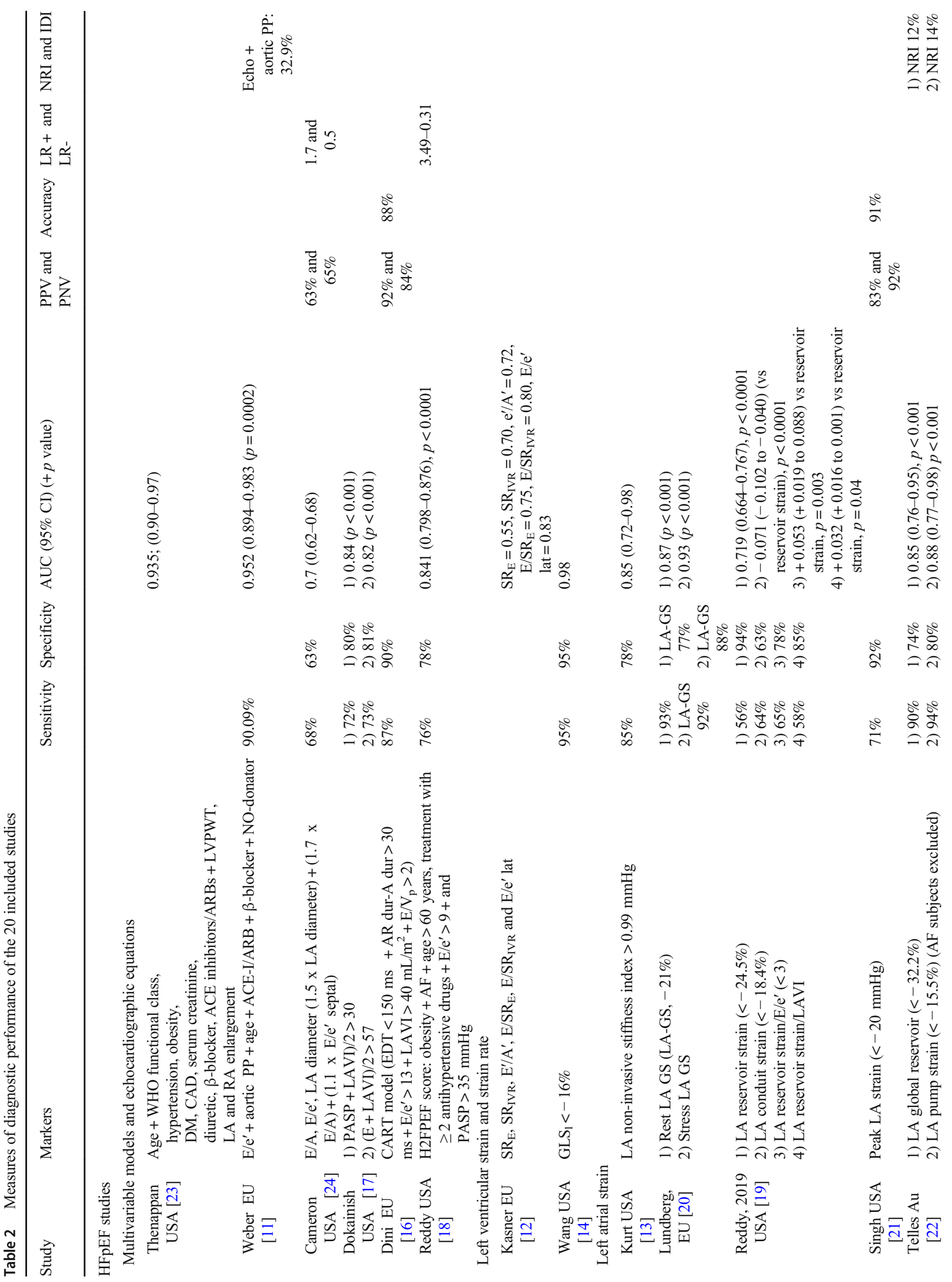




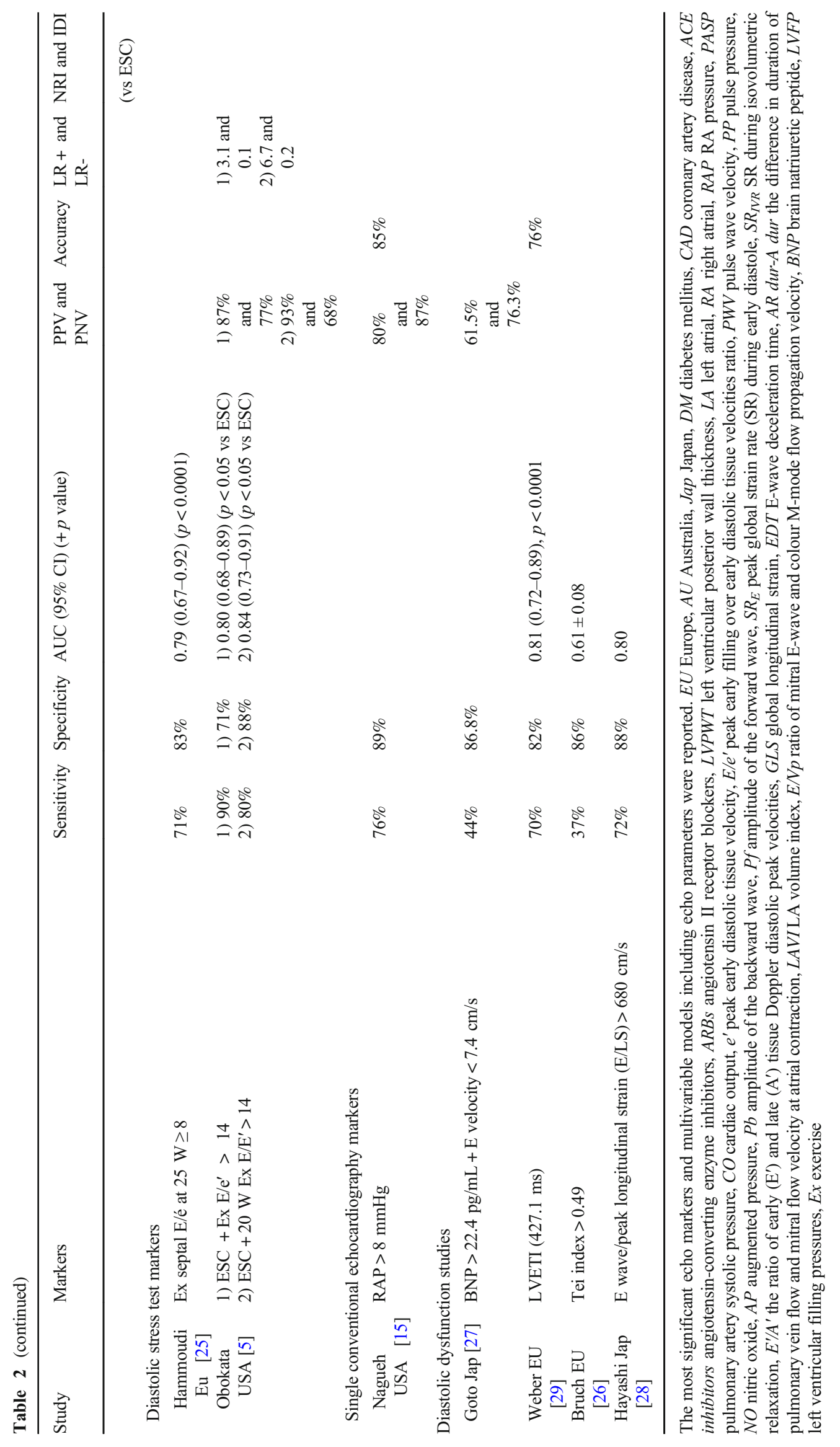


Summary sensitivity of LA strain

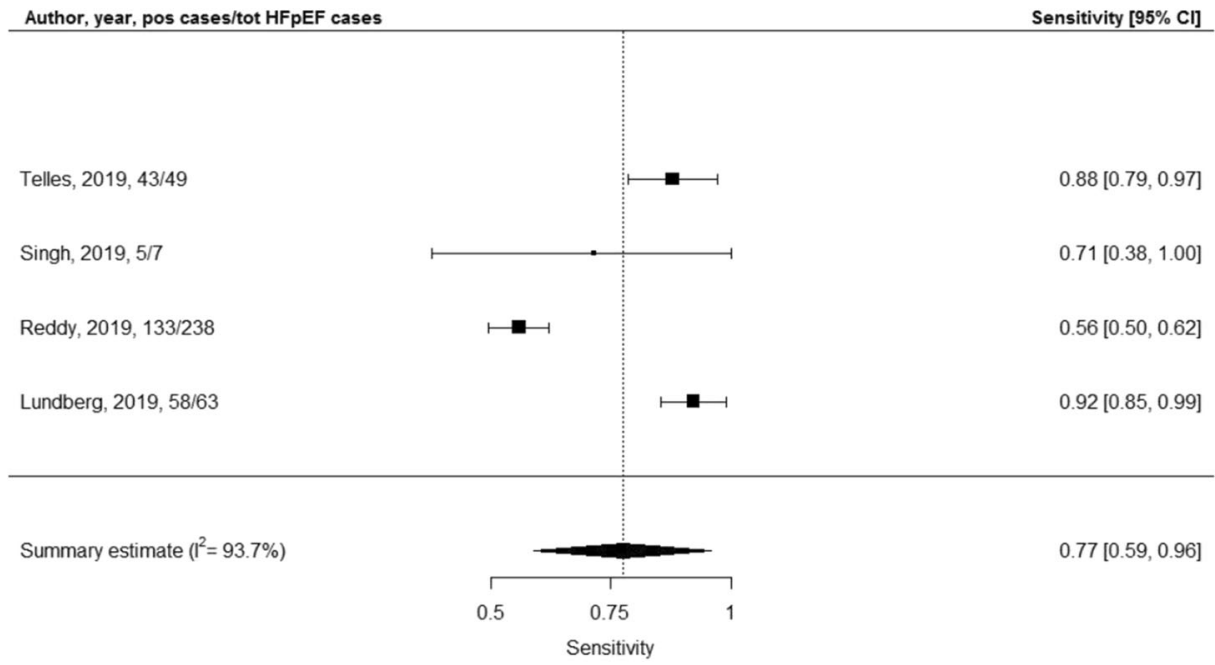

Summary specificity of LA strain

Author, year, neg cases/tot NCD cases

Telles, 2019, 17/22

Singh, 2019, 24/25

Reddy, 2019, 117/125

Lundberg, 2019, 26/29

Summary estimate $\left(l^{2}=91.2 \%\right)$

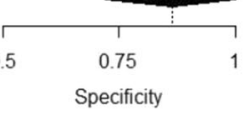

Summary AUC of LA strain

Author, year, pos cases/tot HFpEF cases

Telles, $2019,43 / 49$

Reddy, 2019, 133/238

Lundberg, 2019, 58/63

Summary estimate $\left(l^{2}=88.3 \%\right)$

AUC $[95 \% \mathrm{Cl}]$

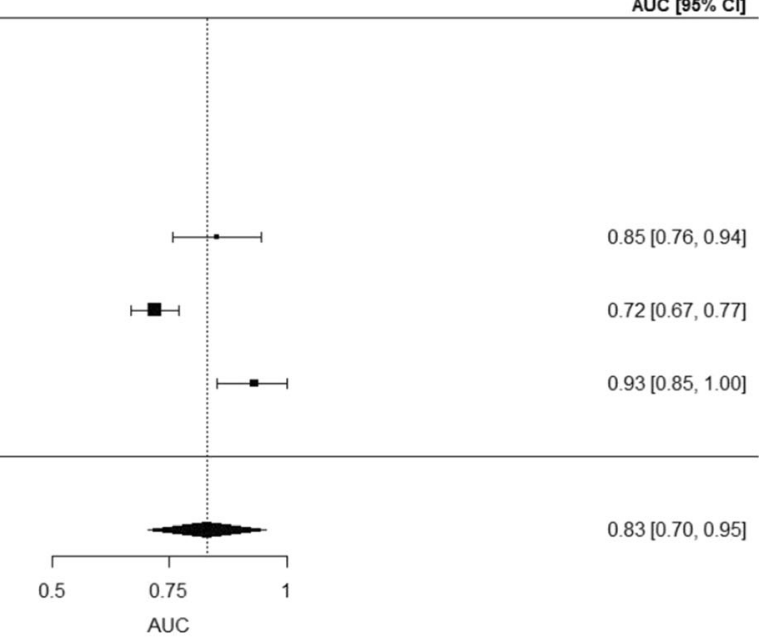


Fig. 1 Meta-analysis of sensitivity, specificity and AUC of LA global strain for the detection of HFpEF with controls with non-cardiac dyspnoea

test (different combinations of echocardiographic techniques and clinical markers). In addition, quality assessment showed a large number of studies with high risk of bias across several domains. For example, 15 of 20 studies excluded subjects not in sinus rhythm. It is well known that HFpEF with concurrent arrhythmias and especially with AF is increasingly common [34]. The exclusion of subjects with AF questions the possibility to efficiently and practically use the newly tested echocardiographic markers in individuals with HFpEF and rhythm abnormalities, limiting generalizability. Another aspect that may have affected the results is the interpretation of the index test, since the echocardiographic analysis was often not blinded from the catheterization or not simultaneous, performed by different investigators, and only in three studies, the cut-off value of the echocardiographic marker was specified before the analysis [11, 22, 24, 25]. This could have resulted in an overestimation of performance of the proposed predictor, questioning its validity.

\section{Conclusions}

In conclusion, despite the considerable heterogeneity of the included studies which does not allow to draw definite conclusion, this study supports an integrated approach for the diagnosis of HFpEF, which includes multiple clinical and echocardiographic measures. New echocardiographic indices such as LA strain and DST data have potential diagnostic value to enhance the detection of HFpEF and LVDD. However, before their implementation into the diagnostic workup, their added diagnostic utility, beyond the established clinical and echocardiographic HFpEF features, should be proven by larger studies of HFpEF versus NCD subjects.

Funding information The research of $\mathrm{SH}$ has received funding from the European Union Commission's Seventh Framework programme under grant agreement N 305507 (HOMAGE), N 602904 (FIBROTARGETS) and N261409 (MEDIA) and N 278249 (EU MASCARA) and the MarieCurie Industry Academy Pathways and Partnerships (CARDIOMIR) N 285991, FP7-Health-2013-Innovations-1 N602156 (HECATOS). It was supported by research grants from the Netherlands Organization for Scientific Research (NWO) Vidi 91796338. This research is cofinanced as a PPP-allowance Research and Innovation by the Ministry of Economic Affairs within Top Sector Life sciences \& Health. We acknowledge the support from the Netherlands Cardiovascular Research Initiative, an initiative with support of the Dutch Heart Foundation, CVON2016-Early HFPEF, 2015-10, and CVON She-PREDICTS, grant 2017-21. Joline WJ Beulens is supported by a ZonMw VIDI grant (91 71 8304).

\section{Compliance with ethical standards}

Conflict of interest The authors declare that they have no conflict of interest.

Open Access This article is licensed under a Creative Commons Attribution 4.0 International License, which permits use, sharing, adaptation, distribution and reproduction in any medium or format, as long as you give appropriate credit to the original author(s) and the source, provide a link to the Creative Commons licence, and indicate if changes were made. The images or other third party material in this article are included in the article's Creative Commons licence, unless indicated otherwise in a credit line to the material. If material is not included in the article's Creative Commons licence and your intended use is not permitted by statutory regulation or exceeds the permitted use, you will need to obtain permission directly from the copyright holder. To view a copy of this licence, visit http://creativecommons.org/licenses/by/4.0/.

\section{References}

1. Vasan RS, Xanthakis V, Lyass A, Andersson C, Tsao C, Cheng S et al (2018) Epidemiology of left ventricular systolic dysfunction and heart failure in the Framingham study: an echocardiographic study over 3 decades. JACC Cardiovasc Imaging 11(1):1-11

2. Ponikowski P, Voors AA, Anker SD, Bueno H, Cleland JG, Coats AJ, Falk V, González-Juanatey JR, Harjola VP, Jankowska EA, Jessup M, Linde C, Nihoyannopoulos P, Parissis JT, Pieske B, Riley JP, Rosano GM, Ruilope LM, Ruschitzka F, Rutten FH, van der Meer P, Authors/Task Force Members, Document Reviewers (2016) 2016 ESC guidelines for the diagnosis and treatment of acute and chronic heart failure: the task force for the diagnosis and treatment of acute and chronic heart failure of the European Society of Cardiology (ESC). Developed with the special contribution of the Heart Failure Association (HFA) of the ESC. Eur J Heart Fail 18(8):891-975

3. Nagueh SF, Smiseth OA, Appleton CP, Byrd BF 3rd, Dokainish H, Edvardsen $\mathrm{T}$ et al (2016) Recommendations for the evaluation of left ventricular diastolic function by echocardiography: an update from the American Society of Echocardiography and the European Association of Cardiovascular Imaging. Eur Heart J Cardiovasc Imaging 17(12):1321-1360

4. Nauta JF, Hummel YM, van der Meer P, Lam CSP, Voors AA, van Melle JP (2018) Correlation with invasive left ventricular filling pressures and prognostic relevance of the echocardiographic diastolic parameters used in the 2016 ESC heart failure guidelines and in the 2016 ASE/EACVI recommendations: a systematic review in patients with heart failure with preserved ejection fraction. Eur $\mathrm{J}$ Heart Fail 20(9):1303-1311

5. Obokata M, Kane GC, Reddy YN, Olson TP, Melenovsky V, Borlaug BA (2017) Role of diastolic stress testing in the evaluation for heart failure with preserved ejection fraction: a simultaneous invasive-echocardiographic study. Circulation 135(9):825-838

6. McMurray JJ, Carson PE, Komajda M, McKelvie R, Zile MR, Ptaszynska A et al (2008) Heart failure with preserved ejection fraction: clinical characteristics of 4133 patients enrolled in the IPRESERVE trial. Eur J Heart Fail 10(2):149-156

7. Pieske B, Tschope C, de Boer RA, Fraser AG, Anker SD, Donal E et al (2020) How to diagnose heart failure with preserved ejection fraction: the HFA-PEFF diagnostic algorithm: a consensus recommendation from the Heart Failure Association (HFA) of the European Society of Cardiology (ESC). Eur J Heart Fail 22(3): $391-412$ 
8. Morris DA, Belyavskiy E, Aravind-Kumar R, Kropf M, Frydas A, Braunauer K, Marquez E, Krisper M, Lindhorst R, Osmanoglou E, Boldt LH, Blaschke F, Haverkamp W, Tschöpe C, Edelmann F, Pieske B, Pieske-Kraigher E (2018) Potential usefulness and clinical relevance of adding left atrial strain to left atrial volume index in the detection of left ventricular diastolic dysfunction. JACC Cardiovasc Imaging 11(10):1405-1415

9. Whiting PF, Rutjes AW, Westwood ME, Mallett S, Deeks JJ, Reitsma JB, Leeflang MM, Sterne JA, Bossuyt PM, QUADAS-2 Group (2011) QUADAS-2: a revised tool for the quality assessment of diagnostic accuracy studies. Ann Intern Med 155(8):529536

10. Ma X, Nie L, Cole SR, Chu H (2016) Statistical methods for multivariate meta-analysis of diagnostic tests: an overview and tutorial. Stat Methods Med Res 25(4):1596-1619

11. Weber T, Wassertheurer S, O'Rourke MF, Haiden A, Zweiker R, Rammer M, Hametner B, Eber B (2013) Pulsatile hemodynamics in patients with exertional dyspnea: potentially of value in the diagnostic evaluation of suspected heart failure with preserved ejection fraction. J Am Coll Cardiol 61(18):1874-1883

12. Kasner M, Gaub R, Sinning D, Westermann D, Steendijk P, Hoffmann W, Schultheiss HP, Tschope C (2010) Global strain rate imaging for the estimation of diastolic function in HFNEF compared with pressure-volume loop analysis. Eur J Echocardiogr 11(9):743-751

13. Kurt M, Wang J, Torre-Amione G, Nagueh SF (2009) Left atrial function in diastolic heart failure. Circ Cardiovasc Imaging 2(1): $10-15$

14. Wang J, Khoury DS, Yue Y, Torre-Amione G, Nagueh SF (2008) Preserved left ventricular twist and circumferential deformation, but depressed longitudinal and radial deformation in patients with diastolic heart failure. Eur Heart J 29(10):1283-1289

15. Nagueh SF, Smiseth OA, Dokainish H, Andersen OS, Abudiab MM, Schutt RC, Kumar A, Gude E, Sato K, Harb SC, Klein AL (2018) Mean right atrial pressure for estimation of left ventricular filling pressure in patients with Normal left ventricular ejection fraction: invasive and noninvasive validation. J Am Soc Echocardiogr 31(7):799-806

16. Dini FL, Ballo P, Badano L, Barbier P, Chella P, Conti U, de Tommasi SM, Galderisi M, Ghio S, Magagnini E, Pieroni A, Rossi A, Rusconi C, Temporelli PL (2010) Validation of an echoDoppler decision model to predict left ventricular filling pressure in patients with heart failure independently of ejection fraction. Eur $\mathbf{J}$ Echocardiogr 11(8):703-710

17. Dokainish H, Nguyen J, Sengupta R, Pillai M, Alam M, Bobek J, Lakkis N (2010) New, simple echocardiographic indexes for the estimation of filling pressure in patients with cardiac disease and preserved left ventricular ejection fraction. Echocardiography 27(8):946-953

18. Reddy YNV, Carter RE, Obokata M, Redfield MM, Borlaug BA (2018) A simple, evidence-based approach to help guide diagnosis of heart failure with preserved ejection fraction. Circulation 138(9): $861-870$

19. Reddy YNV, Obokata M, Egbe A, Yang JH, Pislaru S, Lin G, Carter R, Borlaug BA (2019) Left atrial strain and compliance in the diagnostic evaluation of heart failure with preserved ejection fraction. Eur J Heart Fail 21(7):891-900

20. Lundberg A, Johnson J, Hage C, Back M, Merkely B, Venkateshvaran A et al (2019) Left atrial strain improves estimation of filling pressures in heart failure: a simultaneous echocardiographic and invasive haemodynamic study. Clin Res Cardiol 108(6):703-715

21. Singh A, Medvedofsky D, Mediratta A, Balaney B, Kruse E, Ciszek B, Shah AP, Blair JE, Maffessanti F, Addetia K, Mor-Avi V, Lang RM (2019) Peak left atrial strain as a single measure for the non-invasive assessment of left ventricular filling pressures. Int $\mathrm{J}$ Cardiovasc Imaging 35(1):23-32

22. Telles F, Nanayakkara S, Evans S, Patel HC, Mariani JA, Vizi D, William J, Marwick TH, Kaye DM (2019) Impaired left atrial strain predicts abnormal exercise haemodynamics in heart failure with preserved ejection fraction. Eur J Heart Fail 21(4):495-505

23. Thenappan T, Shah SJ, Gomberg-Maitland M, Collander B, Vallakati A, Shroff P, Rich S (2011) Clinical characteristics of pulmonary hypertension in patients with heart failure and preserved ejection fraction. Circ Heart Fail 4(3):257-265

24. Cameron DM, McLaughlin VV, Rubenfire M, Visovatti S, Bach DS (2017) Usefulness of echocardiography/Doppler to reliably predict elevated left ventricular end-diastolic pressure in patients with pulmonary hypertension. Am J Cardiol 119(5):790-794

25. Hammoudi N, Laveau F, Helft G, Cozic N, Barthelemy O, Ceccaldi A, Petroni T, Berman E, Komajda M, Michel PL, Mallet A, le Feuvre C, Isnard R (2017) Low level exercise echocardiography helps diagnose early stage heart failure with preserved ejection fraction: a study of echocardiography versus catheterization. Clin Res Cardiol 106(3):192-201

26. Bruch C, Schmermund A, Dagres N, Katz M, Bartel T, Erbel R (2002) Tei-index in coronary artery disease-validation in patients with overall cardiac and isolated diastolic dysfunction. Z Kardiol 91(6):472-480

27. Goto T, Ohte N, Wakami K, Asada K, Fukuta H, Mukai S, Tani T, Kimura G (2010) Usefulness of plasma brain natriuretic peptide measurement and tissue Doppler imaging in identifying isolated left ventricular diastolic dysfunction without heart failure. Am J Cardiol 106(1):87-91

28. Hayashi T, Yamada S, Iwano H, Nakabachi M, Sakakibara M, Okada K, Murai D, Nishino H, Kusunose K, Watanabe K, Ishizu T, Wakami K, Yamada H, Dohi K, Seo Y, Ohte N, Mikami T, Tsutsui H (2016) Left ventricular global strain for estimating relaxation and filling pressure- a multicenter study. Circ J 80(5):11631170

29. Weber T, Auer J, O'Rourke MF, Punzengruber C, Kvas E, Eber B (2006) Prolonged mechanical systole and increased arterial wave reflections in diastolic dysfunction. Heart 92(11):1616-1622

30. Borlaug BA, Paulus WJ (2011) Heart failure with preserved ejection fraction: pathophysiology, diagnosis, and treatment. Eur Heart J 32(6):670-679

31. Ikonomidis I, Aboyans V, Blacher J, Brodmann M, Brutsaert DL, Chirinos JA et al (2019) The role of ventricular-arterial coupling in cardiac disease and heart failure: assessment, clinical implications and therapeutic interventions. A consensus document of the European Society of Cardiology Working Group on Aorta \& Peripheral Vascular Diseases, European Association of Cardiovascular Imaging, and Heart Failure Association. Eur J Heart Fail 21(4):402-424

32. Sepehrvand N, Alemayehu W, Dyck GJB, Dyck JRB, Anderson T, Howlett J, Paterson I, McAlister FA, Ezekowitz JA, On behalf of the Alberta HEART Investigators (2019) External validation of the $\mathrm{H} 2 \mathrm{~F}-\mathrm{PEF}$ model in diagnosing patients with heart failure and preserved ejection fraction. Circulation 139(20):2377-2379

33. Santos AB, Kraigher-Krainer E, Gupta DK, Claggett B, Zile MR, Pieske B et al (2014) Impaired left atrial function in heart failure with preserved ejection fraction. Eur J Heart Fail 16(10):1096-1103

34. Goyal P, Almarzooq ZI, Cheung J, Kamel H, Krishnan U, Feldman DN, Horn EM, Kim LK (2018) Atrial fibrillation and heart failure with preserved ejection fraction: insights on a unique clinical phenotype from a nationally-representative United States cohort. Int J Cardiol 266:112-118

Publisher's note Springer Nature remains neutral with regard to jurisdictional claims in published maps and institutional affiliations. 